\title{
NOUVELLE
}

\section{La transdifférenciation cellulaire au service de la sclérose latérale amyotrophique?}

Charles Nicaise, Delphine Bohl, Roland Pochet
C. Nicaise, R. Pochet : Laboratoire d'histologie, neuroanatomie et neuropathologie, Université libre de Bruxelles, Bruxelles, Belgique. D. Bohl : Unité rétrovirus et transfert génétique, Inserm U622, Institut Pasteur, Paris, France. rpochet@ulb.ac.be
La thérapie cellulaire de la sclérose latérale amyotrophique

Paradoxalement, en augmentant l'espérance de vie, nous sommes soumis au défi majeur que constitue l'augmentation exponentielle des maladies neurodégénératives. Parmi celles-ci, la sclérose latérale amyotrophique (SLA) se caractérise par la perte spécifique des neurones moteurs corticaux, bulbaires et spinaux. Les patients atteints de SLA développent des faiblesses musculaires, des fasciculations progressant vers des atrophies plus sévères et des paralysies des muscles périphériques. L'issue fatale survient quand les neurones moteurs innervant le diaphragme et les muscles respiratoires sont touchés. À ce jour, aucun traitement n'existe pour contrecarrer cette disparition massive de neurones. Malgré plus d'un siècle de recul et l'identification de mutations dans les gènes SODI (superoxide dismutase), FUS (fused in sarcoma/translocated-in-liposarcoma) ou TDP43 (TAR DNA-binding protein), rien n'est connu sur la cause originelle de la dégénérescence des neurones moteurs. Cependant, grâce aux modèles animaux transgéniques SODI reproduisant la SLA, plusieurs mécanismes pathologiques menant à la mort des neurones moteurs ont été identifiés: toxicité liée au glutamate, stress oxydatif, formation d'agrégats protéiques, dysfonctionnement des mitochondries et du système autophagique, ou encore pénurie de facteurs neurotrophiques. Il est de plus en plus évident que les cellules non neuronales, astrocytes et microglie, jouent un rôle proactif dans la mort des neurones et dans l'avancée de la maladie, même si elles ne détiennent pas le primum movens dans l'apparition des symptômes $[1,16]$. La SLA est donc une maladie multifactorielle touchant à la fois les cellules neuronales et les cellules non neuronales, et nécessite une approche thérapeutique multicellulaire [2]. La thérapie cellulaire offre de nouveaux espoirs aux patients même si la probabilité de remplacer un neurone déficient par un nouveau neurone capable d'assurer la même fonction et de restaurer les bonnes connexions reste très faible. Remplacer non seulement les neurones moteurs lésés mais aussi les astrocytes par de nouvelles cellules dérivées de cellules souches est une approche qui a été envisagée dès 2007. Fortes des résultats obtenus dans l'étude des modèles animaux SOD1, les thérapies utilisant des cellules dérivées de cellules souches humaines furent rapidement transférées en clinique [3]. Les premiers essais cliniques chez l'homme utilisant des cellules souches mésenchymateuses ou neurales sont en cours d'évaluation en Italie et aux États-Unis [4]. À défaut de remplacer les cellules détruites, la transplantation de cellules neuves peut permettre de prévenir la mort neuronale en fournissant des cellules accessoires et des molécules neuroprotectrices, activant les processus de réparation endogène [2]. La transplantation dans la moelle épinière d'astrocytes sains ou de progéniteurs modifiés pour exprimer différents facteurs neurotrophiques a montré des effet bénéfiques sur la survie des rats SODI et celle des neurones moteurs [5]. Les nouveaux protocoles de reprogrammation épigénétique de cellules humaines adultes en cellules souches pluripotentes induites (iPS, induced pluripotent stem cell) offrent aujourd'hui une source quasi infinie de cellules neuronales ou gliales. Ces iPS, obtenues à partir de cellules somatiques de patients - fibroblastes de peau ou kératinocytes de cheveux par exemple -, peuvent servir autant de modèle d'étude cellulaire de la maladie que de réservoir de cellules thérapeutiques à greffer chez le même patient [6].

\section{La transdifférenciation au service de la SLA ?}

\section{iPS et transdifférenciation}

L'applicabilité clinique des iPS se heurte principalement au temps que prennent la génération, l'amplification et la caractérisation des cellules (2 à 3 mois), à la difficulté de leur différenciation en cellules d'intérêt et au risque de formation tumorale post-transplantation. En effet, si un jour des cellules dérivées d'iPS sont proposées en clinique, il faudra éliminer le risque de contamination de la greffe par des cellules pluripotentes susceptibles de former des tumeurs [7] $\rightarrow)$. Ces limitations peuvent être dorénavant contournées

$(\rightarrow)$ Voir l'article de B. piang et al., page 805 de ce numéro 


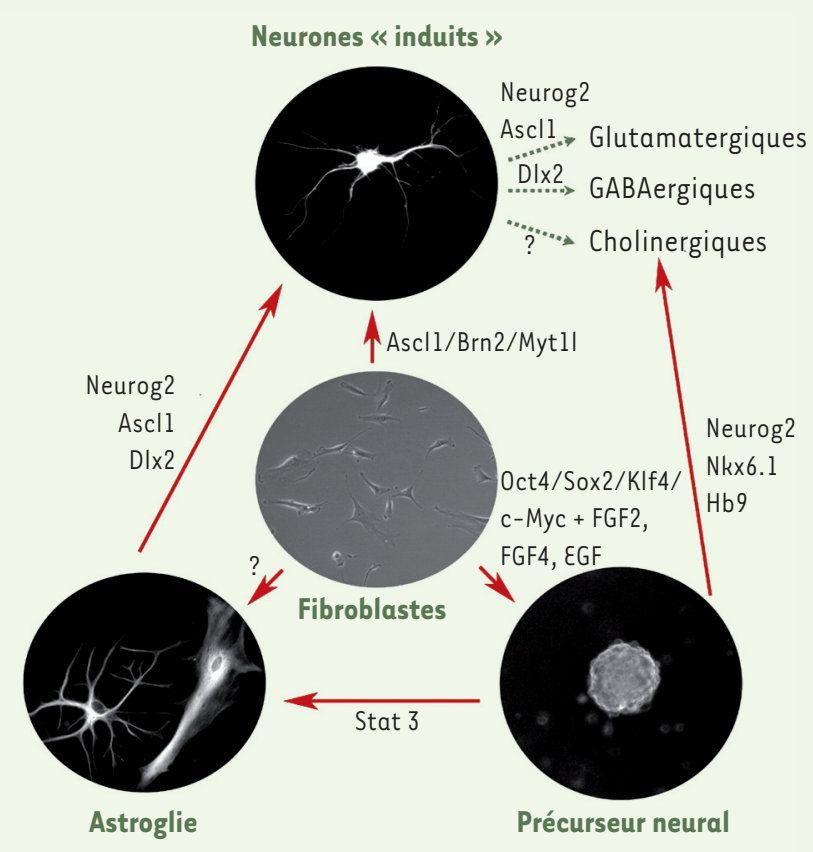

par une stratégie de transdifférenciation, c'est-à-dire de reprogrammation directe d'une cellule différenciée d'une lignée en une cellule différenciée d'une autre lignée, en évitant le stade intermédiaire des iPS (Figure 1). Vierbuchen et al. furent les premiers à décrire la conversion directe et rapide de fibroblastes murins embryonnaires en neurones fonctionnels appelés neurones « induits » [8]. La combinaison de trois facteurs de transcription, Ascll, Brn2 et Mytll impliqués dans la neurogenèse, mais sans rapport avec une propriété de pluripotence contrairement aux facteurs de transcription qui sont utilisés pour une reprogrammation en iPS, transforment in vitro efficacement, en moins d'une semaine, des fibroblastes murins en neurones fonctionnels capables de générer des potentiels d'action (20\% des cellules initiales sont converties). La majorité des neurones ainsi induits était constituée de neurones excitateurs exprimant des marqueurs corticaux. De là il ne restait qu'un pas à franchir pour réaliser cet exploit à partir de cellules humaines. La même équipe vient de montrer que les trois mêmes facteurs de transcription combinés au facteur
NeuroDl convertissent des fibroblastes fœtaux ou postnatals humains en neurones induits [9] (Figure 1). II reste néanmoins à démontrer que le programme fibroblastique est définitivement éteint et à relever le prochain défi : générer directement d'autres sous-types spécifiques de neurones, voire des sous-types de cellules gliales.

\section{Conversion de cellules astrogliales en neurones fonctionnels}

En effet, chez les patients SLA, les celIules touchées sont majoritairement des neurones moteurs de types glutamatergique et cholinergique. Le groupe de Berninger a déjà tracé la route en montrant que la transduction d'un seul facteur de transcription peut convertir des cellules astrogliales post-natales en sous-types neuronaux [10]. Cette conversion n'est pas liée à une plasticité des cellules gliales puisque in vitro ou in vivo il n'est pas possible de transformer des cellules gliales en neurones sans « revenir » à l'étape d'un précurseur neural exprimant des facteurs de détermination neurogénique. II s'agit bel et bien de transdifférenciation cellulaire! L'expression forcée du facteur de
Figure 1. Différents types de cellules neurales obtenus par transdifférenciation et potentiellement utiles au traitement de la SLA. Si la transplantation cellulaire devient une thérapie standard de la SLA, il sera nécessaire de produire in vitro différents types cellulaires pour remplacer les cellules nerveuses déficientes de la moelle épinière. À l'instar de la transmutation chimique, il est possible aujourd'hui de transdifférencier directement des fibroblastes en neurones ou en précurseurs neuraux et ce grâce à des vecteurs rétroviraux exprimant des facteurs de transcription spécifiques. À partir de cellules somatiques post-natales de souris, plusieurs types cellulaires ou sous-types neuronaux ont été créés avec succès : astrocytes, neurones glutamatergiques ou cholinergiques.

transcription Neurog2 dans les cellules astrogliales (via un rétrovirus) conduit à la formation de neurones excitateurs glutamatergiques, alors que l'expression de Dlx2 conduit à la formation de neurones inhibiteurs GABAergiques [11] (Figure 1). Cette équipe a également montré qu'il était possible de générer des neurones fonctionnels par transdifférenciation à partir de cellules astrogliales humaines provenant d'une zone lésée du cerveau, siège d'une gliose réactive.

\section{Reprogrammation directe de cellules somatiques en précurseurs neuraux}

Une approche thérapeutique plus globale de la SLA pourrait tirer parti de la production de précurseurs neuraux, cellules immatures donc en amont des neurones différenciés, pouvant spontanément se différencier in vivo en neurones, astrocytes ou cellules microgliales. Jusqu'à présent, ces précurseurs n'étaient obtenus qu'à partir de tissu fœtal, d'iPS ou de cellules souches embryonnaires, et dans ces deux derniers types de cellules, après culture dans un milieu de culture défini en présence de molécules inhibitrices de la voie de signalisation SMAD, 
telle que Noggin. II est désormais possible de transdifférencier des fibroblastes murins directement en précurseurs neuraux $\mathrm{Pax}^{+}$(et non pas en neurones) en utilisant les quatre mêmes facteurs de transcription (0ct4, Sox2, KIf4, c-Myc) que pour une reprogrammation en iPS (Figure 1). Les seules différences résident dans le contrôle temporel de l'expression des facteurs de transcription exogènes (3 jours seulement) et dans l'exposition à un milieu enrichi en FGF2 (fibroblast growth factor), FGF4 et EGF (epidermal growth factor) en lieu et place du milieu de culture pour cellules souches embryonnaires [12]. Cette procédure est rapide (moins de 2 semaines) et a pour avantage l'expansion in vitro des précurseurs en vue de transplantation à large échelle, alors que les neurones dits induits sont des cellules post-mitotiques et dès lors ne peuvent plus se diviser. Par ailleurs, il est aujourd'hui possible de forcer la différenciation de ces précurseurs neuraux en neurones moteurs de type cholinergique par ingénierie génétique grâce à l'expression des facteurs de transcription $\mathrm{Hb}$, Nkx6.1 et Neurog2 et leur traitement par l'acide rétinoïque et le morphogène sonic hedgehog [13].

Parallèlement, de nombreux progrès en matière de contrôle de la différenciation des précurseurs neuraux en astrocytes ont été réalisés. $\varepsilon$ n effet, étant donné les effets bénéfiques d'astrocytes sains transplantés dans la moelle épinière de rats SOD1, il serait envisageable d'en produire de grandes quantités in vitro. Ces astrocytes jouent un rôle neuroprotecteur dans la moelle épinière des rats SODl en augmentant localement l'expression des transporteurs du glutamate et ils diminuent la toxicité liée au glutamate [5]. On sait maintenant que des signaux extracellulaires comme le FGF2 et le CNTF (ciliary neurotrophic factor) ou encore l'acide rétinoïque et le LIF (leu- kemia inhibiting factor) ont la propriété d'activer, au sein de précurseurs neuraux, le facteur de transcription STAT3, ce qui entraîne un remodelage de la chromatine et des modifications épigénétiques menant finalement à une différenciation astrocytaire [14]. Ces connaissances réunies permettent d'envisager un jour la reprogrammation d'une seule cellule en de multiples types cellulaires nerveux, transplantables chez les patients SLA.

\section{Conclusion}

Le concept de la transplantation cellulaire en thérapie de la SLA a considérablement évolué ces dernières années. Depuis peu, les cellules nerveuses peuvent être reprogrammées, différenciées et exprimer une pléthore de gènes d'intérêt et ainsi améliorer leur intégration, leur survie et délivrer des molécules thérapeutiques (Figure 1). La thérapie cellulaire est une réalité qui a déjà fait ses preuves, notamment pour les maladies du sang. Néanmoins la route est encore longue et le passage des essais sur les rongeurs vers l'humain reste un obstacle qui devra sans doute passer par des modèles comme le chien. Aujourd'hui plus qu'hier et moins que demain on peut sérieusement considérer que la greffe de cellules ou de précurseurs nerveux pourra diminuer la perte des motoneurones et ralentir la progression de la maladie chez les patients atteints de SLA. Le défi est de convaincre les pouvoirs publics et les investisseurs privés d'investir dans des études cliniques où le nombre de patients est suffisamment large. La bataille n'est pas gagnée car une fraction importante de cliniciens neurologues ne considèrent pas cette approche comme efficace [15] et le débat est ouvert. $\diamond$

Cellular transdifferentiation in amyotrophic lateral sclerosis

\section{CONFLIT D’INTÉRÊTS}

Les auteurs déclarent n'avoir aucun conflit d'intérêts concernant les données publiées dans cet article.

\section{RÉFÉRENCES}

1. Boillee S, Lobsiger CS. Les cellules gliales : pas d'un si grand support pour les motoneurones. Med Sci (Paris) $2008 ; 24: 124-6$.

2. Lindvall 0 , Kokaia Z. Stem cells in human neurodegenerative disorders: time for clinical translation? J Clin Invest 2010 ; $120: 29-40$.

3. Hedlund $\varepsilon$, Hefferan MP, Marsala M, Isacson 0. Cell therapy and stem cells in animal models of motor neuron disorders. Eur J Neurosci 2007 ; 26 : 1721-37.

4. Mazzini L, Ferrero I, Luparello V, et al. Mesenchymal stem cell transplantation in amyotrophic lateral sclerosis: a phase I clinical trial. Exp Neurol 2010 ; $223: 229-37$.

5. Lepore AC, Rauck B, Dejea C, et al. Focal transplantation-based astrocyte replacement is neuroprotective in a model of motor neuron disease. Nat Neurosci 2008 ; 11 : 1294-301.

6. Takahashi K, Tanabe K, Ohnuki M, et al. Induction of pluripotent stem cells from adult human fibroblasts by defined factors. Cell 2007 ; $131: 861-72$.

7. Rashid ST, Vallier L. Induced pluripotent stem cells: alchemist's tale or clinical reality? Exp Rev Mol Med $2010 ; 12: 25$.

8. Vierbuchen T, Ostermeier A, Pang ZP, et al. Direct conversion of fibroblasts to functional neurons by defined factors. Nature 2010 ; 463 : 1035-41.

9. Pang ZP, Yang N, Vierbuchen $T$, et al. Induction of human neuronal cells by defined transcription factors. Nature $2011 ; 476: 220-3$.

10. Berninger B, Costa MR, Koch U, et al. Functional properties of neurons derived from in vitro reprogrammed postnatal astroglia. J Neurosci 2007 ; $27: 8654-64$.

11. Heinrich C, Blum R, Gascon S, et al. Directing astroglia from the cerebral cortex into subtype specific functional neurons. PLoS Biol 2010 ; 8 : e1000373.

12. Kim J, Efe JA, Zhu S, et al. Direct reprogramming of mouse fibroblasts to neural progenitors. Proc Natl Acad Sci USA 2011 ; 108 : 7838-43.

13. Bohl D, Liu S, Blanchard S, et al. Directed evolution of motor neurons from genetically engineered neural precursors. Stem Cells 2008 ; 26 : 2564-75.

14. Asano H, Aonuma M, Sanosaka T, et al. Astrocyte differentiation of neural precursor cells is enhanced by retinoic acid through a change in epigenetic modification. Stem Cells $2009 ; 27$ : 2744-52.

15. Fornai F, Meininger V, Silani V. Future therapeutical strategies dictated by pre-clinical evidence in ALS. Arch Ital Biol $2011 ; 149$ : 169-74.

16. Dupuis $L$, Loeffler JP. Sclérose latérale amyotrophique, jonction neuromusculaire et déficit énergétique. Med Sci (Paris) 2008 ; 24 : 1077-82. 